\title{
Extended donor criteria in lung transplantation: Impact on organ allocation
}

\author{
Phil Botha, MRCS, ${ }^{a}$ Dipesh Trivedi, FRCS, ${ }^{a}$ Christopher J. Weir, PhD, ${ }^{b}$ Cait P. Searl, FRCA, ${ }^{a}$ Paul A. Corris, FRCP, \\ John H. Dark, FRCS, and Stephan V. B. Schueler, FRCS ${ }^{a}$
}

From the Department of Cardio-pulmonary Transplantation, Freeman Hospital, ${ }^{\mathrm{a}}$ Newcastle upon Tyne, United Kingdom; the Robertson Centre for Biostatistics, University of Glasgow, ${ }^{\mathrm{b}}$ Glasgow, United Kingdom; and the Immunology and Transplantation Research Group, University of Newcastle upon Tyne, ${ }^{\mathrm{c}}$ Newcastle upon Tyne, United Kingdom.

Received for publication Sept 16, 2005; revisions received Nov 25, 2005; accepted for publication Dec 9, 2005.

Address for reprints: Phil Botha, MRCS, Department of Cardiopulmonary Transplantation Freeman Hospital, High Heaton, Newcastle upon Tyne, NE7 7DN, United Kingdom (E-mail: P.Botha@ncl.ac.uk).

J Thorac Cardiovasc Surg 2006;131:1154-60

$0022-5223 / \$ 32.00$

Copyright () 2006 by The American Association for Thoracic Surgery

doi:10.1016/j.jtcvs.2005.12.037
Objective: Some reports have documented a higher early mortality with the use of extended criteria donors in lung transplantation. None have evaluated how outcomes compare with the use of these organs for single and bilateral transplantation or whether this practice results in a higher incidence of early bronchiolitis obliterans syndrome.

Methods: We performed a retrospective review of case notes, intensive therapy unit database, and donor details. Between January 1, 2000, and December 31, 2004, 201 patients underwent lung or heart-lung transplantation.

Results: Eighty-three (41.3\%) patients received organs deemed marginal on the basis of at least one of the following criteria: donor age greater than 55 years, duration of ventilation greater than 5 days, purulent secretions or inflammation at bronchoscopy, smoking of 20 or more cigarettes per day, abnormality on chest roentgenogram, or $\mathrm{Po}_{2} /$ fraction of inspired oxygen ratio of less than $300 \mathrm{~mm} \mathrm{Hg}$ immediately before donor organ procurement. Recipients of marginal lungs had a higher incidence of severe (grade 3) primary graft dysfunction $(43.9 \%$ vs $27.4 \%, P$ $=.015)$ and 90 -day organ-specific mortality $(15.7 \%$ vs $5.1 \%, P=.012)$. Bilateral transplantation carried a significantly higher 30-day mortality if performed with marginal organs $(17.0 \%$ vs $2.7 \%$ with standard donor organs, $P=.005)$. Thirty-day mortality was not significantly different for the transplantation of single marginal or standard donor lungs. Cumulative survival and survival free of bronchiolitis obliterans syndrome was not affected by marginal donor status.

Conclusion: Transplantation of extended criteria donor lungs leads to a higher incidence of primary graft dysfunction. Bilateral transplantation with these organs seems to confer less reserve, resulting in a higher early mortality rate. Medium-term functional outcome is, however, not adversely affected by the relaxation of donor criteria.

$\mathrm{A}$ $\mathrm{t}$ least $14 \%$ of patients on the waiting list for lung transplantation in the United Kingdom continue to die each year. A further $2 \%$ of patients are removed from the list because of deterioration in their clinical condition. ${ }^{1}$ In the subgroup of patients with fibrotic lung disease, up to $40 \%$ die before transplantation in many series. ${ }^{2,3}$ Donor organ shortage thus remains one of the foremost causes of death for patients with end-stage lung disease eligible for transplantation. Several methods of expanding the current donor pool have been suggested, with the relaxation of previously strict donor criteria and use of donor organs previously deemed unsuitable or "marginal" being used in many centers. Optimization of donor management has also received much interest because this has resulted in higher procurement rates. ${ }^{4}$ Despite these measures, only $18 \%$ of brainstem-dead organ donors in the United Kingdom have lungs suitable for transplantation. ${ }^{1}$

Several authors have evaluated the use of marginal donors in their own centers, with conflicting reports documenting either identical outcomes to those with standard donors or, in some cases, greater risk of early mortality. ${ }^{5,6}$ To date, donor data 


\author{
Abbreviations and Acronyms \\ $\mathrm{BLT}=$ bilateral lung transplantation \\ BOS $=$ bronchiolitis obliterans syndrome \\ $\mathrm{CPB}=$ cardiopulmonary bypass \\ $\mathrm{CXR}=$ chest roentgenogram \\ $\mathrm{FIO}_{2}=$ fraction of inspired oxygen \\ ITU $=$ intensive therapy unit \\ PGD $=$ primary graft dysfunction \\ SLT $=$ single lung transplantation
}

are only collected by individual centers, and no centralized database exists. As a result, there are no validated criteria for designating donors as marginal, and criteria used have differed from center to center. This has undoubtedly affected outcome analysis. Some have questioned how the use of marginal organs for higher-risk recipients affects transplantation outcome as a whole. ${ }^{5}$ None have evaluated specifically how outcomes differ in single lung transplantation (SLT) versus bilateral lung transplantation (BLT) with marginal organs. Published views support the use of marginal organs for BLT, a practice that reflects opinion and not an evidence base.

These factors bear an important influence on decision making in transplantation, namely which recipients are suitable for marginal lung allocation without conferring an unacceptable survival disadvantage. With lung transplantation imparting disparate survival benefits to patients with different diagnoses, the appropriate allocation of organs from marginal donors should be made with concrete outcome data to support it. In an attempt to answer some of these questions, we retrospectively collected patient and donor characteristics, as well as outcome data, for all lung transplantations performed at our center over a 5-year period and investigated associations between marginal donor characteristics and several measures of outcome.

\section{Patients and Methods}

Data for all adult (age $\geq 16$ years) lung and heart-lung transplant recipients at the Freeman Hospital during the period from January 1,2000 , to December 31,2004, were collected retrospectively by means of review of clinical notes, the intensive care database, and donor information sheets.

\section{Marginal Donors}

Organ assessment at the time of procurement included review of available chest roentgenograms (CXR), flexible bronchoscopy, inspection and palpation of the thoracic cavity and lungs, and measurement of differential pulmonary vein gasses. Additional factors assessed in identifying marginal donors included age, smoking history, and duration of ventilation. Donors were considered marginal if they met any of the following criteria: age greater than 55 years, current smoking of 20 or more cigarettes per day, duration of ventilation of longer than 5 days, radiographic infiltrates in the lung to be trans- planted, purulent secretions or inflammation at bronchoscopy, arterial $\mathrm{Po}_{2}$ of less than $40 \mathrm{kPa}(300 \mathrm{~mm} \mathrm{Hg}$ ) on $100 \%$ inspired oxygen, and $5 \mathrm{~cm} \mathrm{H} \mathrm{H}_{2} \mathrm{O}$ of positive end-expiratory pressure at last measurement before donor pneumonectomy.

\section{Lung Allocation and Transplantation}

Lung allocation in the United Kingdom is based on zones. If no suitable recipient exists within the zonal transplant center, the organ or organs are offered to extraregional centers through the national organ allocation network. Offers are made to centers on a sequential basis, determined by when they last accepted an organ from the national pool. Significant autonomy exists within the centers to allocate offered organs to an appropriate recipient on the basis of blood group, size match, urgency, diagnosis, and time on the waiting list.

As of April 2001, we have used a single flush technique with Perfadex (Vitrolife Göteborg, Sweden) and side-table retrograde flush with the same solution for organ preservation. Other zonal centers have used alternative preservation methods, including Euro-Collins or Papworth solutions or core cooling, during the study period. Lung transplantation is performed with single lung ventilation or cardiopulmonary bypass $(\mathrm{CPB})$ where indicated in single lungs. CPB is used as a routine for all bilateral sequential transplants. Reperfusion is performed at controlled pressure with unaltered blood.

\section{Early and Late Graft Dysfunction}

Primary graft dysfunction (PGD) scoring was performed retrospectively on the basis of gas exchange data derived from the intensive care notes and radiologist reports of postoperative CXRs. Scoring was performed according to the International Society for Heart and Lung Transplantation working classification for PGD as follows 7 : grade 0 , no abnormality on $\mathrm{CXR}$ and $\mathrm{PO}_{2} /$ fraction of inspired oxygen $\left(\mathrm{FIO}_{2}\right)$ ratio greater than $300 \mathrm{~mm} \mathrm{Hg}$; grade 1, infiltrates on CXR consistent with reimplantation edema and $\mathrm{PO}_{2} /$ $\mathrm{FIO}_{2}$ ratio greater than $300 \mathrm{~mm} \mathrm{Hg}$; grade 2, CXR infiltrates and $\mathrm{Po}_{2} / \mathrm{FIO}_{2}$ ratio less than $300 \mathrm{~mm} \mathrm{Hg}$ and greater than $200 \mathrm{~mm} \mathrm{Hg}$; and grade 3, CXR infiltrates and $\mathrm{PO}_{2} / \mathrm{FIO}_{2}$ ratio less than $200 \mathrm{~mm}$ Hg. PGD scores were calculated for values at arrival in the intensive care unit and 24, 48, and 72 hours after transplantation. Maximum grade of PGD attained during the first 72 hours after transplantation was used for analysis. Alveolar-arterial oxygen gradient was calculated by using the alveolar gas equation at arrival in the intensive care unit and 24 hours later.

Bronchiolitis obliterans syndrome (BOS) was defined according to International Society for Heart and Lung Transplantation guidelines. ${ }^{8}$ Grade 1 BOS (decrease in forced expiratory volume in 1 second of more than $20 \%$ from baseline value) was used as onset of BOS, and early onset of BOS was defined as onset within 1 year of transplantation.

\section{Statistical Considerations}

Unless otherwise stated, continuous data are presented as means \pm standard deviation and analyzed by using a 2-sample $t$ test. Variables with large standard deviations relative to the mean indicating a skewed distribution (eg, duration of ventilation and intensive care unit stay) were analyzed with the Mann-Whitney test. Categoric data are presented as counts followed by percentages in 
TABLE 1. Recipient demographics

\begin{tabular}{lc}
\hline Recipient demographics & $\mathbf{N}=\mathbf{2 0 1}$ \\
\hline Age, y & $39.99 \pm 14.1$ \\
Male sex & $95(47.3 \%)$ \\
Diagnosis & \\
Suppurative & $84(42.0 \%)$ \\
Obstructive & $69(34.5 \%)$ \\
Fibrotic & $27(13.5 \%)$ \\
PPH & $10(5.0 \%)$ \\
Other & $10(5.0 \%)$ \\
\hline
\end{tabular}

$P P H$, Primary pulmonary hypertension.

parentheses and analyzed by using the $\chi^{2}$ test. Categoric survival outcomes were 30- and 90-day mortality and organ-specific mortality at 90 days. Cumulative survival and freedom from BOS were calculated by using the Kaplan-Meier method, and groups were compared by using the log-rank test. All hypothesis tests used a $5 \%$ significance level. Statistical analysis was performed with SPSS version 12.0.1 software.

\section{Results}

In the 5-year period studied, 201 lung and heart-lung transplantations were performed. Of these, $62(30.8 \%)$ were SLTs, $122(60.7 \%)$ were BLTs, and 17 (8.5\%) were heartlung transplantations. The most frequent recipient diagnosis was suppurative lung disease (84 [41.8\%]), whereas obstructive lung disease (69 [34.5\%]) and fibrotic lung disease (27 [13.5\%]) accounted for most of the other diagnoses. Recipient demographics are listed in Table 1. Eighty-three (41.3\%) of the donors met at least one marginal criterion. Of these, $58(28.9 \%)$ met 1 criterion, $19(9.5 \%)$ met 2 criteria, and $6(3.0 \%)$ met 3 criteria. Donor demographics are listed in Table 2.

\section{TABLE 2. Donor demographics}

\begin{tabular}{lc}
\hline Donor demographics & $\mathbf{N}=\mathbf{2 0 1}$ \\
\hline Age, y & $36.7 \pm 13.7$ \\
Male sex & $94(46.8 \%)$ \\
Cause of death & \\
Cerebrovascular event & $118(58.7 \%)$ \\
Head injury & $56(27.9 \%)$ \\
Infective & $12(6.0 \%)$ \\
Neoplastic & $4(2.0 \%)$ \\
Other & $11(5.5 \%)$ \\
Marginal criteria & \\
Age $>55$ y & $17(8.5 \%)$ \\
Ventilated $>5$ days & $19(9.5 \%)$ \\
Smoking $\geq 20 / d$ & $32(15.9 \%)$ \\
CXR infiltrates & $21(10.4 \%)$ \\
Abnormal bronchoscopy & $14(7.0 \%)$ \\
Po ${ }_{2}<300$ mm Hg & $11(5.5 \%)$ \\
Donors meeting 1 or more criteria & $83(41.3 \%)$ \\
\hline
\end{tabular}

CXR, Chest roentgenogram.

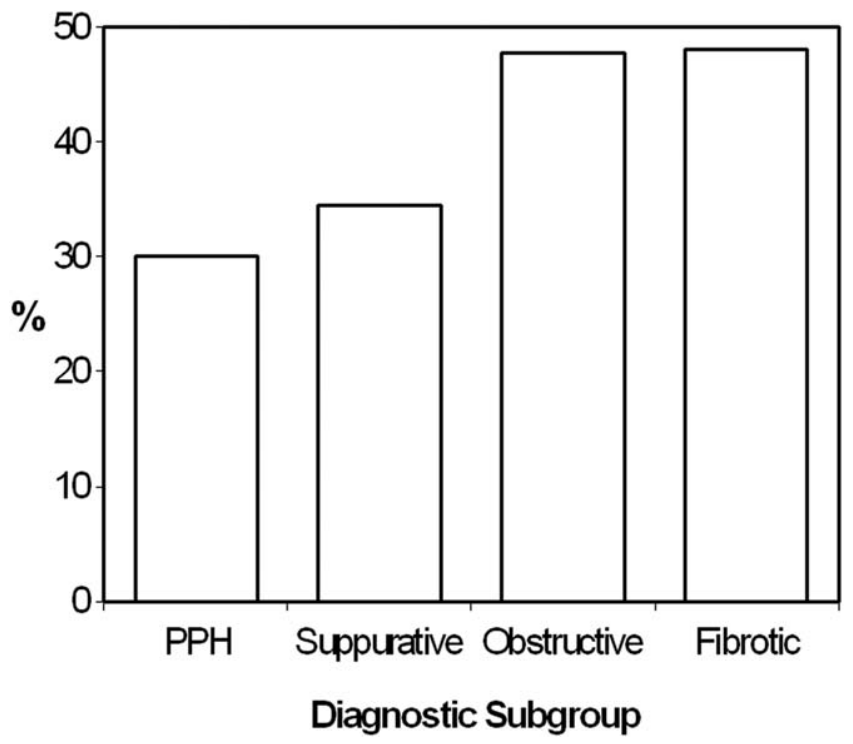

Figure 1. Allocation of marginal organs: percentage of patients in each diagnostic subgroup receiving organs from marginal donors. PPH, Primary pulmonary hypertension.

\section{Organ Allocation}

In the 201 lung and heart-lung transplantations analyzed, proportionally more SLTs were performed with marginal organs $(32[51.6 \%])$. Bilateral transplantations were performed with marginal organs in $51(36.7 \%)$ patients $(P=$ .47). The allocation of standard organs favored patients in the primary pulmonary hypertension and suppurative disease (predominantly cystic fibrosis) subgroups, whereas slightly fewer patients with obstructive lung disease and fibrotic lung disease received standard donor lungs $(P=$ .36 , Figure 1). Recipients of lungs from marginal donors had a higher mean age $(43.4 \pm 14$ vs $37.6 \pm 13.8$ years, $P=.004)$.

\section{Postoperative Graft Function}

Ischemic time was $317 \pm 66$ minutes for the group as a whole and not statistically different for those receiving marginal or standard lungs $(319 \pm 65$ and $316 \pm 67$ minutes, respectively; $P=.76$ ). Severe (grade 3) PGD occurred in $68(34.2 \%)$ of all transplantations performed. Differential outcomes for recipients receiving standard versus marginal donor lungs are listed in Table 3 . On inspection of the differential outcome data at various durations of ischemic time, those longer than 405 minutes led to higher rates of grade 3 PGD in recipients of marginal lungs (83.3\% vs $40.8 \%, P=0.043)$. In recipients of standard lungs, this influence of prolonged ischemic time was not observed (30.0\% vs $27.1 \%$ grade 3 PGD, $P=0.844$ ).

Duration of positive pressure ventilation (median, 29 vs 29 hours; $95 \%$ confidence interval for difference, -7.0 to 
TABLE 3. Outcomes in recipients of standard donor lungs compared with marginal donor lungs

\begin{tabular}{|c|c|c|c|}
\hline Outcomes & Standard donor & Marginal donor & $P$ value \\
\hline Grade 3 PGD & $32(27.4 \%)$ & $36(43.9 \%)$ & .015 \\
\hline $\begin{array}{l}\text { Mean Aa gradient } \\
\text { at } 24 \mathrm{~h}\end{array}$ & $115 \pm 80 \mathrm{~mm} \mathrm{Hg}$ & $148 \pm 118 \mathrm{~mm} \mathrm{Hg}$ & .021 \\
\hline $\begin{array}{l}\text { Median duration } \\
\text { of ventilation }\end{array}$ & $29 \mathrm{~h}$ & $29 \mathrm{~h}$ & .76 \\
\hline Median ITU stay & $2 d$ & $3 d$ & .93 \\
\hline 30-d mortality & $10(8.5 \%)$ & $13(15.7 \%)$ & .12 \\
\hline 90-d mortality & $15(12.7 \%)$ & $19(22.9 \%)$ & .058 \\
\hline $\begin{array}{l}\text { Organ-specific } \\
\text { mortality (90 d) }\end{array}$ & $6(5.1 \%)$ & $13(15.7 \%)$ & .012 \\
\hline BOS at $1 \mathrm{y}$ & $13(11 \%)$ & $4(4.8 \%)$ & .12 \\
\hline
\end{tabular}

$P G D$, Primary graft dysfunction; Aa gradient, alveolar-arterial oxygen gradient; $I T U$, intensive therapy unit; $B O S$, bronchiolitis obliterans syndrome.

8.0 hours; $P=.76$ ) and intensive therapy unit (ITU) stay (median, 3 vs 2 days; $95 \%$ confidence interval for difference, -1.0 to 1.0 days; $P=.93$ ) were not significantly different for marginal and standard donor lungs. Thirty-day mortality for the entire cohort was $11.4 \%(\mathrm{n}=23)$, whereas 90-day mortality was $16.9 \%(\mathrm{n}=34)$. As demonstrated in Table 3,30- and 90-day mortalities were not significantly different among recipients of standard and marginal donor lungs. Organ-specific mortality at 90 days (respiratory failure or multiorgan failure with severe PGD) was, however, significantly higher in recipients of marginal lungs (13 [15.7\%] compared with 6 [5.1\%] in the standard donor group, Table 3).

\section{Differential Outcome}

SLT carried a significantly higher 30-day mortality than BLT (12 [19.4\%] vs 10 [8.2\%], $P=.033)$. After heart-lung transplantation, the 30-day mortality was $5.7 \%$. In the 62 recipients of a single lung transplant, 30-day mortality did not differ significantly between those receiving marginal and standard organs $(4 / 32[12.5 \%]$ vs $8 / 30$ [26.7\%], $P=$
.158). In recipients of BLTs, however, the use of marginal donor organs significantly affected 30-day mortality. Recipients of bilateral marginal donor lungs had an unexpectedly high 30-day mortality of $17.0 \%$ (8/47), whereas the 30-day mortality was low $(2.7 \%$ [2/75]) in recipients of 2 standard lungs $(P=.005$, Figure 2$)$. At 90 days, this difference in mortality rates remained statistically significant for patients undergoing BLT $(P=.008)$ and nonsignificant for patients undergoing $\operatorname{SLT}(P=.47)$.

Thirty- and 90-day mortality rates were $18.5 \%$ and $29.6 \%$ for fibrotic lung disease, $15.9 \%$ and $23.2 \%$ for obstructive lung disease, and $7.1 \%$ and $9.5 \%$ for suppurative lung disease, respectively. SLT for obstructive lung disease carried a statistically nonsignificant tendency for greater risk of 30-day mortality (21.4\%) than sequential lung transplantation for the same indication $(4.0 \%, P=.053)$. The size of the cohort undergoing transplantation for fibrotic lung disease also prevented meaningful statistical analysis. When outcome of marginal organ use was analyzed for different diagnostic subgroups, only those undergoing transplantation for suppurative lung disease had significant differences in outcome. Because of the obligatory use of BLT in this group, 30-day mortality was similarly high $(17.2 \%)$ for those receiving marginal organs and very low $(1.8 \%)$ for recipients of standard organs $(P=$ $.009)$.

Survival was not statistically different for all-cause mortality $(P=.28, \log$-rank test; Figure 3$)$ in the 2 groups. Similarly, organ-specific mortality was not statistically significantly different for standard and marginal lungs $(P=$ .23, log-rank test). The incidence of early-onset BOS (within 1 year of transplantation) was not statistically different for the 2 groups, at $11.0 \%$ in recipients of standard donor organs and $4.8 \%$ in recipients of marginal lungs $(P=$ .12). BOS-free survival was also calculated by using the Kaplan-Meier method and was not statistically significantly different between marginal and standard transplants $(P=$ .85 , log-rank test; Figure 4).

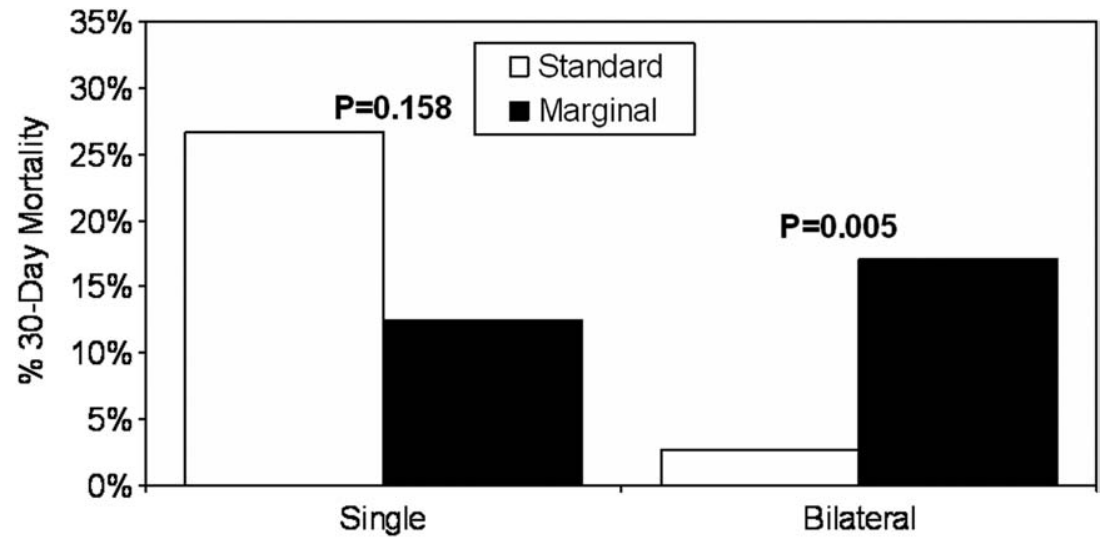

Figure 2. Thirty-day mortality with marginal and standard donors in single and bilateral transplantation. 


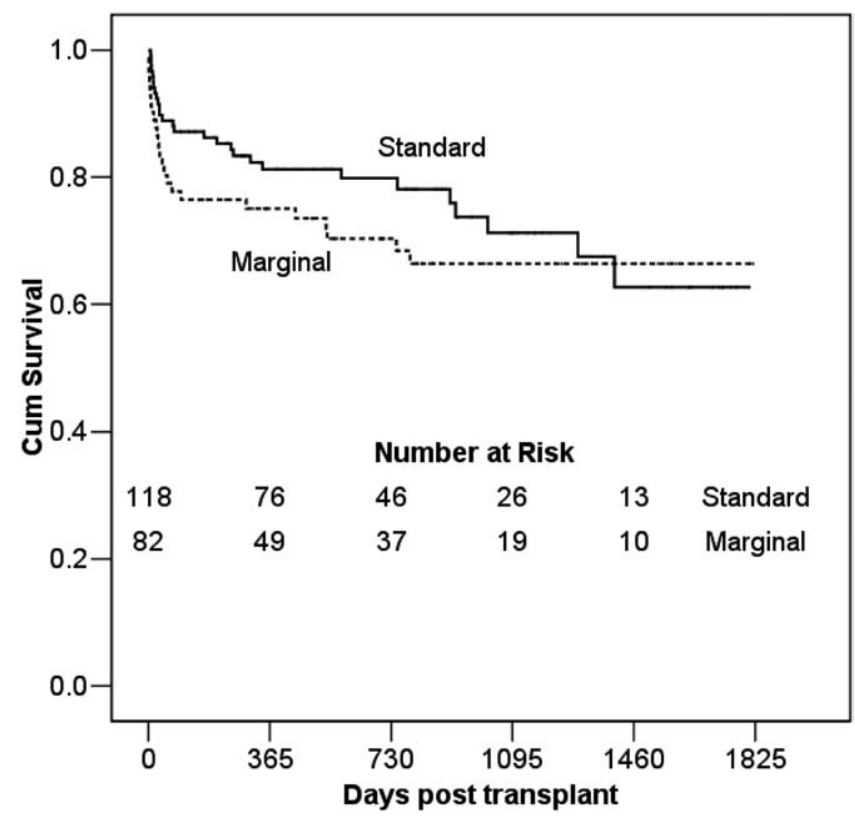

Figure 3. Cumulative survival (all-cause mortality) for recipients of standard versus marginal donor lungs (in days). $P=.28$, log-rank test.

\section{Discussion}

Since the first report of successful relaxation of donor criteria with acceptable outcome by Kron and colleagues, ${ }^{9}$ several centers have reported encouraging results in this regard. In the absence of a validated set of criteria for defining donors as marginal, the definition of the marginal/ extended donor has varied, making comparison between these reports difficult. In the largest series to date, Gabbay and associates ${ }^{4}$ reported 140 transplants from 112 donors, with $64(57 \%)$ not meeting all criteria for standard lung donors. Their marginal donors included $20(31 \%)$ patients with low $\mathrm{PO}_{2} / \mathrm{FIO}_{2}$ ratios at referral, which improved to acceptable levels with manipulation. They also used a large number (39) of organs with radiographic infiltrates and 24 donor lungs with infection, as defined by purulent secretions or Gram staining. These organs were accepted if ischemic time would not be extended (beyond 6 hours); the recipient was unlikely to undergo transplantation otherwise and required BLT. SLT was performed only where clear unilateral donor lung dysfunction existed. They found no significant difference in gas exchange, ITU stay, and early mortality with the use of these lungs. Straznicka and colleagues ${ }^{10}$ reported no difference in outcome with the use of marginal organs that included a large group of more than $51 \%$ in whom a previous low $\mathrm{Po}_{2} / \mathrm{FIO}_{2}$ ratio or CXR abnormality had been corrected by means of donor management. They also showed no difference in survival with the use of standard or extended criteria lungs when recipient diagnosis was

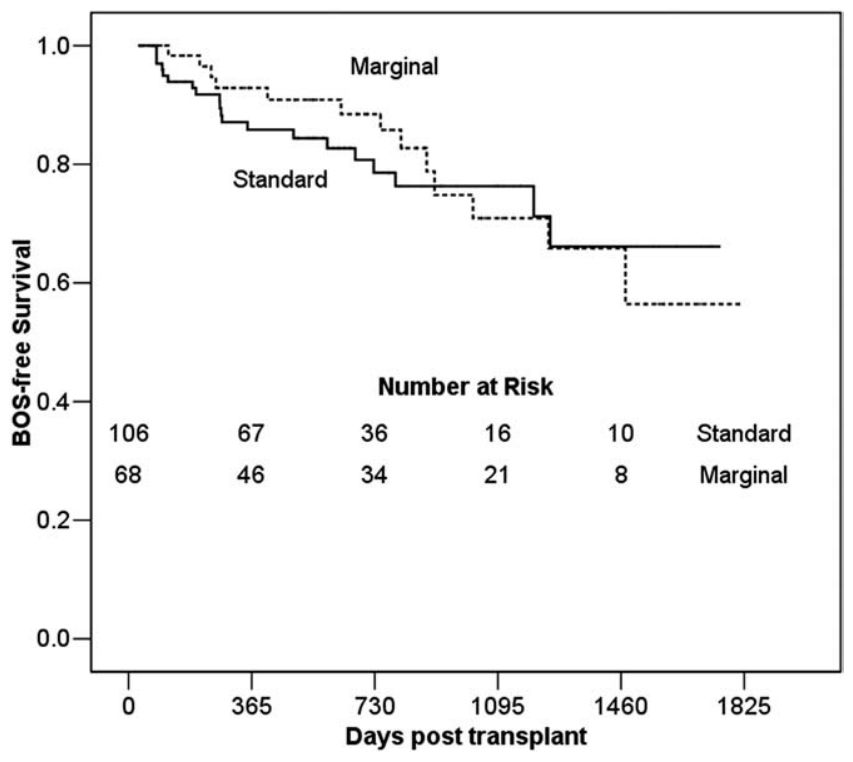

Figure 4. Cumulative bronchiolitis obliterans syndrome (BOS)free survival (in days). $P=.85$, log-rank test.

entered into a Cox proportional hazards model. The Toronto group was the first to document a higher early mortality with the use of marginal donor organs. ${ }^{5}$ They especially cautioned against the use of marginal organs in high-risk recipients. They demonstrated a higher mortality (22.2\%) when these organs were used for transplantation in patients of advanced age or with Burkholderia cepacia colonization before transplantation. Our experience has confirmed their findings, showing a higher early organ-specific mortality with the use of marginal donor lungs.

Sundaresan and colleagues ${ }^{11}$ documented no difference in early outcome between recipients of standard and marginal organs but found that CPB was required more frequently for implantation of the second lung in BLT with marginal organs (20\%). They postulated that some reversible lung dysfunction might be better tolerated by patients with emphysema and avoided the use of marginal organs in patients with fibrotic lung disease. Bhorade and coworkers ${ }^{12}$ found no difference in hospital survival but demonstrated a statistically nonsignificant difference in spirometry of single marginal lung recipients. They therefore cautioned against the use of single lungs from extended donors. In our cohort, recipients of bilateral marginal lungs had a significantly higher 30-day mortality than those receiving 2 lungs from a standard donor. The criteria used to define our cohort might play a significant role in this finding because a higher proportion of our patients failed at least one criterion affecting the function of both lungs (ie, age, smoking, duration of ventilation, and low total $\mathrm{Po}_{2} / \mathrm{FIO}_{2}$ ratio before donor pneumonectomy). ${ }^{13} \mathrm{We}$ postulated that current heavy 
smoking would have more effect on early graft function than a long smoking history. This was confirmed by the finding of a significantly higher alveolar-arterial oxygen gradient 24 hours after transplantation and longer ITU stay in those receiving lungs from heavy smokers $(179 \pm 111$ $\mathrm{mmHg}$ vs $122 \pm 95 \mathrm{mmHg}, P=.01$, and median ITU stay of 5 vs 2 days). Although the method of preservation has differed from center to center and over time within our study population, a subset analysis revealed a similar distribution of known preservation techniques among marginal and standard donor lungs. The incidence of PGD was slightly higher for non-Perfadex preservation methods, but this difference did not reach statistical significance (results not shown). Our routine use of CPB for BLTs might play a role in aggravating PGD in the marginal lungs. Although previous studies have shown no difference in outcome with the use of CPB for BLT, ${ }^{14}$ we postulate that marginal donor lungs might be more vulnerable to the injurious effects of $\mathrm{CPB}$. We continue to support the use of routine CPB for all BLTs because of the absolute hemodynamic stability and controlled pressure reperfusion it affords. We are, however, investigating the amelioration of injury caused by bypassactivated leukocytes through depletion or pharmacologic means to minimize reperfusion-related injury.

The finding of greater early mortality with BLT with marginal lungs has significant implications for the allocation of donor organs. In the only subgroup in which BLT is mandatory (ie, those with suppurative lung disease), the use of marginal donor lungs results in a significant increase in early mortality. One possible solution might be to allocate (circumstances allowing) these organs predominantly to those with obstructive disease where either single or bilateral transplantation can be performed. Other series have shown no difference in early mortality with SLT or BLT for obstructive disease ${ }^{15}$ but some improvement in late function with BLT. ${ }^{16}$ We prefer to allocate only organs deemed near ideal to those with cystic fibrosis and pulmonary hypertension. Because of the high waiting list mortality, ${ }^{2,17}$ and absence of proved survival benefit with BLT, ${ }^{18}$ single marginal lungs will, of necessity, continue to be used in those with fibrotic lung disease.

In this analysis we have demonstrated a higher rate of PGD and early lung-specific mortality with the use of marginal donor lungs. In keeping with our previous findings, the higher incidence of early allograft damage did not result in a higher incidence of early-onset BOS or poorer BOS-free survival. ${ }^{19}$ Longer follow-up will be needed to exclude a higher lifetime risk of BOS. Our finding of significantly higher 30-day mortality with the use of marginal donor lungs for BLT has significant implications for allocation strategy. The obvious factor that might account in part for this finding is our routine use of CPB for bilateral sequential transplantations. This might aggravate ischemia-reperfusion injury in marginal lungs with limited reserve and influence direct comparison between the groups. Notably, other centers have documented a higher necessity for the use of CPB when marginal lungs are implanted sequentially. ${ }^{11}$ It would nonetheless be interesting to see whether our findings would be duplicated in centers in which BLT is performed routinely without the use of $\mathrm{CPB}$.

\section{References}

1. UK Transplant in the United Kingdom 2003-2004. UK Transplant, Bristol, UK. 2004. ISSN 1741-6949.

2. Thabut G, Mal H, Castier Y, Groussard O, Brugiere O, MarrashChahla R, et al. Survival benefit of lung transplantation for patients with idiopathic pulmonary fibrosis. J Thorac Cardiovasc Surg. 2003; 126:469-75

3. Hosenpud JD, Bennett LE, Keck BM, Edwards EB, Novick RJ. Effect of diagnosis on survival benefit of lung transplantation for end-stage lung disease. Lancet. 1998;351:24-7.

4. Gabbay E, Williams TJ, Griffiths AP, MacFarlane LM, Kotsimbos TC, Esmore DS, et al. Maximizing the utilization of donor organs offered for lung transplantation. Am J Respir Crit Care Med. 1999; 160:265-71.

5. Pierre AF, Sekine Y, Hutcheon MA, Waddell TK, Kheshavjee SH. Marginal donor lungs: a reassessment. J Thorac Cardiovasc Surg. 2002;123:421-8.

6. Lardinois D, Banysch M, Korom S, Hillinger S, Rousson V, Boehler A, et al. Extended donor lungs: eleven years experience in a consecutive series. Eur J Cardiothorac Surg. 2005;27:762-7.

7. Christie JD, Carby M, Bag R, Corris P, Hertz M, Weill D. Report of the ISHLT Working Group on Primary Lung Graft Dysfunction. Part II: Definition-consensus statement of the International Society for Heart and Lung Transplantation. J Heart Lung Transplant. 2005;24: $1454-9$.

8. Estenne MM, Boehler A, Egan JJ, Frost A, Hertz M, Mallory GB, et al. Bronchiolitis obliterans syndrome 2001: an update of the diagnostic criteria. J Heart Lung Transplant. 2002;21:297-310.

9. Kron I, Tribble C, Kern J, Daniel T, Rose C, Truwit J, et al. Successful transplantation of marginally acceptable thoracic organs. Ann Surg. 1993;217:518-24.

10. Straznicka M, Follette DM, Eisner MD, Roberts PF, Menza RL, Babcock WD. Aggressive management of lung donors classified as unacceptable: excellent recipient survival one year after transplantation. J Thorac Cardiovasc Surg. 2002;124:250-8.

11. Sundaresan S, Semenkovich J, Ochoa L, Richardson G, Trulock E, Cooper J, et al. Successful outcome of lung transplantation is not compromised by the use of marginal donor lungs. J Thorac Cardiovasc Surg. 1995;109:1075-80.

12. Bhorade SM, Vigneswaran W, McCabe MA, Garrity EA. Liberalization of donor criteria may expand the donor pool without adverse consequence in lung transplantation. J Heart Lung Transplant. 2000; 19:1200-4.

13. Puskas JD, Winton TL, Miller JD, Scavuzzo M, Patterson GA. Unilateral donor lung dysfunction does not preclude successful contralateral single lung transplantation. J Thorac Cardiovasc Surg. 1992;103: 1015-8.

14. Szeto WY, Kreisel D, Karakousis GC, Pochettino A, Sterman DH, Kotloff RM, et al. Cardiopulmonary bypass for bilateral sequential lung transplantation in patients with chronic obstructive pulmonary disease without adverse effect on lung function and clinical outcome. J Thorac Cardiovasc Surg. 2002;124:241-9.

15. Charman SC, Sharples LD, McNeil KD, Wallwork J. Assessment of survival benefit after lung transplantation by patient diagnosis. $J$ Heart Lung Transplant. 2002;21:226-32.

16. Sundaresan RS, Shiraishi Y, Trulock EP, Manley J, Lynch J, Cooper JD, et al. Single or bilateral lung transplant for emphysema? J Thorac Cardiovasc Surg. 1996;112:1485-95. 
17. De Meester J, Smits JM, Persijn GG, Haverich A. Listing for lung transplantation: life expectancy and transplant effect, stratified by type of end-stage lung disease, the Eurotransplant experience. J Heart Lung Transplant. 2001;20:518-24.

18. Meyers BF, Lynch JP, Trulock EP, Guthrie T, Cooper JD, Patterson GA. Single versus bilateral lung transplantation for idiopathic pulmo- nary fibrosis: a ten-year institutional experience. J Thorac Cardiovasc Surg. 2000;120:99-107.

19. Fisher AJ, Wardle J, Dark JH, Corris PA. Non-immune acute graft injury after lung transplantation and the risk of subsequent bronchiolitis obliterans syndrome (BOS). J Heart Lung Transplant. 2002;21: 1206-12.

\section{Online-www.aats.org}

Now you can get The Journal of Thoracic and Cardiovascular Surgery online. The Journal online brings you faster delivery time, easy searching of current and back issues, links to PubMed, AATS, WTSA, and other important sites, and more. Visit the Journal online today.

\section{Receive tables of contents by e-mail}

To receive the tables of contents by e-mail, sign up through our Web site at http://journals.elsevierhealth.com/periodicals/ymtc

Choose E-mail Notification

Simply type your e-mail address in the box and click the Subscribe button. You will receive an e-mail to confirm that you have been added to the mailing list.

Note that TOC e-mails will be sent out when a new issue is posted to the Web site. 